Coeliac disease and risk of tuberculosis

\section{Coeliac disease and risk of tuberculosis: a population-based cohort study}

\section{S Sanders, J West, M K B Whyte}

\section{Are patients with coeliac disease at higher risk of tuberculosis?}

O $\mathrm{n}$ the basis of screening studies on healthy volunteers, coeliac disease is now recognised to affect $1 \%$ of the population. ${ }^{1}$ The current optimal serological testing for coeliac disease may be immunoglobin (Ig)A endomysial, IgA tissue transglutaminase or a combination of the two, with a positive predictive value in excess of $95 \%$ provided IgA deficiency is excluded. Patients with positive serology then require an endoscopic duodenal biopsy to confirm the presence of villous atrophy and thus a firm diagnosis of coeliac disease. A link between coeliac disease and tuberculosis was first suggested as far back as 1952, but there has been a paucity of data exploring this relationship other than case reports and case series. ${ }^{2}$ The initial focus was on gastrointestinal tuberculosis being one of the differential diagnoses for villous atrophy of the small bowel. However, in 1988 a report from Birmingham, UK, suggested that patients with coeliac disease seemed to have an increased prevalence of tuberculosis (6/76 patients with coeliac disease reporting a history of tuberculosis ). ${ }^{3}$

The Swedish study reported in this edition of Thorax explores this overlap/ relationship in greater detail. Ludvigsson et $a l^{4}$ (see $p$ 23) investigated the risk of tuberculosis in 14335 patients with coeliac disease and made comparisons against 69888 matched individuals in a general population-based cohort study. Patients with coeliac disease were reported to have an increased risk of subsequent tuberculosis (HR 3.74, 95\% CI 2.14 to $6.53, \mathrm{p}<0.001$ ). This epidemiological study is fairly robust and reflects the strengths of medical data collection in Sweden as a result of the Swedish National In-Patient Register. The findings of this study are, however, limited by the fact that only 24 actual patients with coeliac disease (24/14 335) developed tuberculosis. In the context of a large database study, this observation is a positive statistical finding but identifies a small increase in absolute risk. A caveat, however, is that patients with tuberculosis were ascertained by hospitalisation for this condition, so that some clinically relevant cases might be missed. Importantly, Ludvigsson et al ${ }^{4}$ have looked for an association of coeliac disease with only one respiratory condition rather than many. In the past others have explored the positive associations between coeliac disease and a variety of respiratory conditions such as sarcoidosis and pulmonary fibrosis. ${ }^{5-12}$ This study would thus have been strengthened by study of other infectious and inflammatory lung diseases, such as pneumonia for example, that may not be associated. This would support the view of a mechanistic basis for an association between coeliac disease and tuberculosis, and also persuade readers that the observed association is not simply due to ascertainment bias, that is, that individuals admitted to hospital for one condition, coeliac disease, may then acquire additional diagnoses more frequently than their control population. The authors make rather a leap of faith from finding an association to assuming it is causal and then speculating on a mechanism.

If their finding is indeed a causal coeliac disease be more susceptible to tuberculosis? The authors speculate that this may be related to malabsorption of vitamin D. Vitamin D was recently shown to upregulate antimicrobial peptides such as LL-37, which are implicated in intracellular killing of Mycobacterium tuberculosis. ${ }^{13}$ Vitamin D can also induce nitric oxide synthase in macrophages, which again may suppress growth of $M$ tuberculosis. ${ }^{14}$ An alternative possibility is that the HLA-DQ2 haplotype, which is strongly linked to coeliac disease, is associated with specific alleles of HLA class I and II molecules as well as with genes for tumour necrosis factor (TNF) $\alpha$ and complement factors $\mathrm{C} 2$ and $\mathrm{C} 4$. TNF $\alpha$ polymorphisms may regulate host responses in tuberculosis, although coeliac disease is associated with the TNF308A promoter polymorphism that may protect against tuberculosis. ${ }^{15}$ The $\mathrm{C} 2$ and C4 molecules are also polymorphic and, as the authors suggest, perhaps a variant relationship, why should patients with
C2 allele may promote tuberculosis infection in a subgroup of patients. ${ }^{16}$ Further investigation of these complex haplotypes could provide biological evidence of the relationship between coeliac disease and tuberculosis, or further insight into the pathogenesis of either condition. At a clinical level, a serological study on the prevalence of coeliac disease in patients with tuberculosis might also serve to establish whether there is a relationship between these two disease processes.

How does this study change clinical practice? The findings would be unlikely to lower the threshold for investigating patients with coeliac disease for tuberculosis, given the relatively small number of actual cases reported. What can perhaps be inferred from this study is that, if a relationship between coeliac disease and tuberculosis is confirmed in further studies, then clinicians should consider serological testing for coeliac disease in those patients with tuberculosis who have gastrointestinal symptoms or apparent drug resistance. ${ }^{17}$

Thorax 2007;62:1-2.

doi: $10.1136 /$ thx.2006.062158

\section{Authors' affiliations}

D S Sanders, Gastroenterology and Liver Unit, Royal Hallamshire Hospital, Sheffield, UK J West, Division of Epidemiology and Public Health, University of Nottingham Medical School, Queen's Medical Centre, Nottingham, UK

M K B Whyte, School of Medicine and Biomedical Sciences, University of Sheffield, Sheffield, UK

Correspondence to: Dr D S Sanders, Roya Hallamshire Hospital, Glossop Road, Room P39 P Floor, Sheffield S10 2JF, Sheffield, UK; david. sanders@sth.nhs.uk

Competing interests: None declared.

\section{REFERENCES}

1 West J, Logan RF, Hill PG, et al. Seroprevalence, correlates and characteristics of undetected coeliac disease in England. Gut 2003;52:960-5.

2 Williams $\mathbf{H}$. Coeliac syndrome due to tuberculosis enteritis. Med J Aust 1952;2:388-9.

3 Williams AJ, Asquith $P$, Stableforth DE. Susceptibility to tuberculosis in patients with coeliac disease. Tubercle 1988;69:267-74.

4 Ludvigsson JF, Wahlstrom J, Grunewald J, et al. Coeliac disease and risk of tuberculosis: a population based cohort study. Thorax 2006;62:23-8

5 Berrill WT, Eade OE, Macleod WM, et al. Proceedings: Bird fancier's lung and coeliac disease. Gut 1975;16:825-6.

6 Eade OE, Berrill WT. Coeliac lung disease. Lancet 1978;1:917-18.

7 Muers MF, Faux JA, Ting A, et al. HLA-A, B, C and HLA-DR antigens in extrinsic allergic alveolitis (budgerigar fancier's lung disease). Clin Allergy 1982;12:47-53.

8 Karlish AJ. Coeliac disease and diffuse lung disease. Lancet 1971;1:1077. 
9 Douglas JG, Gillon J, Logan RF, et al. Sarcoidosis and coeliac disease: an association? Lancet 1984;2:13-15.

10 Anon. Sarcoidosis and coeliac disease. Lancet 1984;2:408.

11 Lowe G, Johnston RN. Sarcoidosis and coeliac disease. Lancet 1984;2:637.

12 Rutherford RM, Brutsche MH, Kearns $M$, et al. Prevalence of coeliac disease in patients with sarcoidosis. Eur J Gastroenterol Hepatol 2004;16:911-15.

13 Zasloff M. Fighting infections with vitamin D. Nat Med 2006; 12:388-96.

14 Rockett KA, Brookes R, Udalova I, et al. 1,25Dihydroxyvitamin D3 induces nitric oxide synthase and suppresses growth of Mycobacterium tuberculosis in a human macrophage-like cell line. Infect Immun, 1998 Nov, 66:5314-21.
15 Correa PA, Gomez LM, Cadena J, et al. Autoimmunity and tuberculosis. Opposite association with TNF polymorphisms. J Rheumatol 2005;32:219-24.

16 Schorey JS, Carroll MC, Brown EJ. A macrophage invasion mechanism of pathogenic mycobacteria. Science 1997;277:1091-3.

17 Shetty A, McKendrick M. TB and coeliac disease. $J$ Infect 2004:48:109-11.

\section{High-grade lesion: what does it tell us?}

\section{E S Edell}

\section{Surveillance programmes for patients with high-grade lesions, which may progress to invasive cancer, will require tissue archives for histology and cooperation and collaboration of participating centres}

L ung cancer remains the leading cause of death due to cancer in both men -and women in several countries. Most patients with lung cancer present with non-specific symptoms such as cough, dyspnoea or haemoptysis and have latestage disease at the time of diagnosis. Treatment strategies are thus often palliative rather than curative. Early detection trials using routine chest roentgenograms and sputum cytology have shown that cancers can be detected at earlier stages but the overall mortality from lung cancer was not improved. With new technology developments, such as low-dose computed tomography and fluorescence bronchoscopy, comes new hope that early detection may in fact change this pattern.

Squamous cell carcinoma, although no longer the most common type of lung cancer, remains a major problem, accounting for $17-29 \%$ of lung cancers. ${ }^{1}$ Studies on sputum cytology showed that $11 \%$ of individuals with moderate dysplasia and 19-46\% with severe dysplasia progressed to squamous cell cancers. Once detected, localisation of these early lesions was not easy using conventional white-light bronchoscopy. Thus, fluorescence bronchoscopy systems were developed to improve the detection of preinvasive and invasive lesions of the central airways. Several studies showed the improved detection of both preinvasive and invasive cancers using fluorescence bronchoscopy compared with white-light bronchoscopy alone. ${ }^{2-5}$ As often happens, however, many new questions have surfaced as more patients are found to have these preinvasive lesions.
For example, what should be done with a patient who has an abnormal area showing carcinoma in situ on a biopsy specimen? Several studies have suggested that the carcinoma in situ, if left alone, will progress to invasive cancer and should be treated. ${ }^{67}$ What about a patient with severe dysplasia? Here the data suggest that the rate of progression to invasive cancer is much less but sufficiently high for these patients to be followed-up. ${ }^{8}$ But do we really know the natural history of these lesions?

In this issue of Thorax, George et al ${ }^{10}$ (see $p$ 43) have presented their work on 22 patients with either previous lung cancer, asbestos exposure or known chronic obstructive pulmonary disease. Fifty one lesions were identified using fluorescence bronchoscopy. High-grade lesions included seven classified as severe dysplasia, 28 as carcinoma in situ and 16 as low-grade lesions of mild or moderate dysplasia. These patients were followed up for a median of 23 months (range 1285 months). Indolence or regression was observed in $83 \%$ of the high-grade lesions and no progression was seen in any of the low-grade lesions. However, five patients with high-grade lesions subsequently developed cancers that were detected by computed tomography during their follow-up.

What can be learned from the work by George et al? ${ }^{10}$ Their data do not change the current practice that patients with carcinoma in situ should receive treatment. Surgical resection remains the standard treatment, but local treatment such as photodynamic therapy or argon plasma coagulation could be an option if carried out in a clinical trial. ${ }^{11}$ For patients who are not surgical candidates either because of poor pulmonary function or other comorbidities, local treatment has been shown to be a reasonable option. ${ }^{12-14}$ What about those patients with severe dysplasia? The experience presented by George et al ${ }^{10}$ shows that these patients are at high risk for developing an invasive cancer. In this population, surveillance is important with both fluorescence bronchoscopy and with computed tomography of the chest.

As we look to the future many gaps remain in our understanding of the natural history of squamous cell carcinoma, and lung cancer in general. Studies such as the one by George et $a l^{10}$ have again shown that there are subsets of patients with high-grade lesions that progress to invasive cancers and others that do not. This shows the need for reliable markers in tumour biology. George et $a l^{10}$ point out that surveillance programmes need to include tissue archives of blood, sputum and bronchial specimens, thus enabling studies on histologically identical lesions with different clinical outcomes. This work will require the cooperation and collaboration of many centres involved in surveillance programmes.

Thorax 2007:62:2-3.

doi: 10.1136/thx.2006.067876

Correspondence to: Dr E S Edell, Mayo Clinic, First Avenue SW, Rochester, MN 55902, USA; eedell@mayo.edu

Competing interests: None declared.

\section{REFERENCES}

1 Greenlee RT, Hill-Harmon MB, Murray T, et al. Cancer Statistics, 2001. CA Cancer J Clin 2001;51:15-36.

2 Venmans BJW, van Boxem AJM, Smit EF, et al. Early detection of preinvasive lesions in high-risk patients. A comparison of conventional flexible and fluorescence bronchoscopy. J Bronchol 1998;5:280-3.

3 Horvath T, Horvathova M, Salaika F, et al. Detection of bronchial neoplasia in uranium miners by autofluorescence bronchoscopy. Diagn Ther Endosc 1999:5:91-8.

4 Shibuya K, Fujisawa $\mathrm{T}$, Hoshino $\mathrm{H}$, et al. Fluorescence bronchoscopy in the detection of preinvasive bronchial lesions in patients with sputum cytology suspicious or positive for malignancy. Lung Cancer 2001;32:19-25.

5 Ikeda N, Hiyoshi T, Kakihana M, et al. Histopathological evalutation of fluorescence 


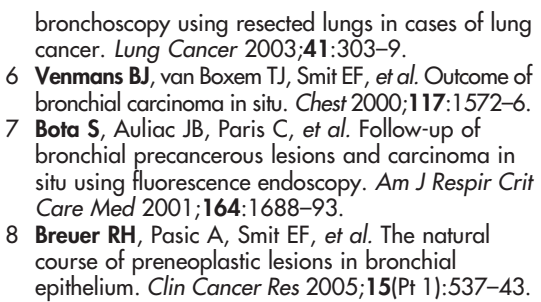

bronchoscopy using resected lungs in cases of lung cancer. Lung Cancer 2003:41:303-9.

6 Venmans BJ, van Boxem TJ, Smit EF, et al. Outcome of bronchial carcinoma in situ. Chest 2000;1 17:1572-6.

7 Bota S, Auliac JB, Paris C, et al. Follow-up of bronchial precancerous lesions and carcinoma in situ using fluorescence endoscopy. Am J Respir Crit Care Med 2001; 164:1688-93.

8 Brever RH, Pasic A, Smit EF, et al. The natural course of preneoplastic lesions in bronchial epithelium. Clin Cancer Res 2005;15(Pt 1):537-43.

9 Lam S, Slivinskas J, McWilliams A, et al. Natural history of premalignant bronchial lesions: implications for chemoprevention. Am Assoc Cancer Res 2002.

10 George PJ, Banerjee AK, Read CA, et al. Surveillance for the detection of early lung cancer in patients with bronchial dysplasia. Thorax 2005;62:43-50.

11 Cortese DA, Edell ES, Kinsey J. Photodynamic therapy for early stage squamous cell carcinoma of the lung. Mayo Clin Proc 1997;72:595-602.
12 Edell ES, Cortese DA. Bronchoscopic phototherapy with hematoporphyrin derivative for treatment of localized bronchogenic carcinoma: a 5 -year experience. Mayo Clin Proc 1987:62:8-14

13 Kato $\mathrm{H}$. Photodynamic therapy for lung cancer of $-\mathrm{a}$ review of 19 years experience. J Photchem Photobiol 1998:42:96-9.

14 Mathur PN, Edell ES, Sutedja T, et al. Treatment of early stage non-small cell lung cancer. Chest 2003;123:S176-80.

\section{What is this thing called CFA?}

\section{A U Wells, D M Hansell, A G Nicholson}

\section{The term "cryptogenic fibrosing alveolitis"should now be used as strictly synonymous with "idiopathic pulmonary fibrosis"}

\footnotetext{
A rcane diagnostic labels bother clinicians. The diffuse lung disease lexicon is a notorious example. For decades, "diffuse lung disease speak" consisted of an unholy mix of histopathological and clinical terms, varying between countries, within countries and even between medical teams in the same hospital. Radical change was required and proposals were advanced in a joint American Thoracic Society and European Respiratory Society (ATS/ERS) initiative. Terminology for idiopathic interstitial pneumonia was distilled by a core group of clinicians, radiologists and pathologists, and this was then circulated to a larger group of international reviewers and published in 2002. ${ }^{1}$ The final consensus classification was not, at first sight, straightforward to apply. Indeed, the amorphous entity of "non-specific interstitial pneumonia" (NSIP) continues to vex clinicians and requires further subclassification. However, teething problems aside, the ATS/ERS initiative has been an outstanding success. Clinicians and researchers worldwide now understand each other better than before. The recent move towards large multicentre treatment studies in idiopathic pulmonary fibrosis (IPF), itself a revolution in slow motion, was made possible, in no small part, by this standardisation of terminology and disease definitions.

As the terminology has changed, the article by Rudd et $a l^{2}$ in the current issue of Thorax (see $p$ 67) is likely to pose difficulties for some non-UK readers. The authors have studied "cryptogenic fibrosing alveolitis" (CFA) as a clinical presentation, as used historically in the UK. The diagnostic criteria, consisting of
}

compatible radiographic, pulmonary function and clinical findings, in the absence of an overt environmental or autoimmune cause, are highly non-specific: compatible, also, with idiopathic interstitial pneumonias other than IPF and a subgroup of patients with hypersensitivity pneumonitis. By contrast, in the ATS/ERS classification, ${ }^{13}$ CFA is explicitly synonymous with IPF, as defined at biopsy or using high resolution computed tomography (HRCT) criteria, with typical clinical features also required, and this is now accepted internationally. Rudd et $a l^{2}$ discuss this issue in their methods section. However, their definition of CFA, as corresponding to "idiopathic pulmonary fibrosis in US terminology", is no longer correct using the current classification and, in reality, was probably never correct. In historical US series, the diagnosis of IPF was wholly or largely based on histopathological data; the application of ATS criteria essentially involved reclassifying NSIP cases and a small number of other disorders. ${ }^{4}$ By contrast, the historical UK entity of CFA was diagnosed at surgical biopsy in only $12 \%$ of cases in the early 1990s, ${ }^{5}$ and a large number of disorders other than IPF were necessarily included. Thus, the current British Thoracic Society (BTS) study is nothing more or less than a study of a non-specific clinical presentation, and unless this is understood, the findings will be misinterpreted.

The presence of two very different entities in the current medical literature, both termed CFA, cannot be a good thing. However, in their use of historical terminology, Rudd et al ${ }^{2}$ perhaps imply that something nosologically important has been lost. The concept of a "CFA" presentation is undoubtedly useful. The typical clinical picture is readily recognised in the outpatient clinic, even before tests such as HRCT. A "CFA" presentation is a key starting point in the personal diagnostic algorithms of many experienced clinicians. Furthermore, knowledge of a clinical picture of "CFA" informs prognostic evaluation. It is increasingly clear that the histological pattern of NSIP is associated with several distinct clinicoradiological entities, with HRCT and clinical findings often those of organising pneumonia with fibrosis ${ }^{6}$ or hypersensitivity pneumonia. ${ }^{7}$ However, the good outcome in these subgroups contrasts with outcomes in patients with idiopathic NSIP with a "CFA" clinical presentation: although prolonged survival is sometimes attainable, a poor IPF-like outcome is equally common, despite aggressive treatment. ${ }^{8}$ A frank admission that the prognosis is uncertain is crucial if therapeutic options are to be discussed with patients in an informed manner.

Perhaps more importantly, a "CFA" presentation has been, and is, indispensable for epidemiological work. It is simply unrealistic to require the application of formal ATS/ERS criteria for IPF, including HRCT, biopsy and bronchoalveolar lavage findings, in studies of the prevalence and spectrum of disease outside referral centres. An inclusive approach is required-in other words, a clinical diagnosis is necessary. Epidemiological work on diffuse lung disease is vital. The track record of public funding bodies (such as the Medical Research Council) in supporting therapeutic studies of diffuse lung disease is poor, partly because of a lingering perception that IPF is an uncommon disease confined to referral centres. Studies to establish the true impact of diffuse lung disease outside specialist units are particularly important in disorders that are strikingly more prevalent with increasing age, but this view was, perhaps, underrepresented in the ATS/ERS deliberations. The redefinition of CFA as synonymous with IPF has effectively disenfranchised epidemiologists, who, it could be argued, now have to study a disorder without a 
name. It is hardly surprising that some researchers in the field are disenchanted with the ATS/ERS classification and cling to the terminology of the past century.

However, the needs of epidemiologists are sometimes trumped by clinical imperatives. Diagnostic separations are valuable when they provide useful distinctions in the natural history and treated course of disease. The ATS/ERS consensus classification passes this test with flying colours: fundamentally, diagnosis is prognosis. In a study of consecutive patients with a "CFA" presentation during the 1980s, who were considered to have CFA at biopsy, reclassification resulted in three broad patient groups. ${ }^{8}$ Histological patterns of usual interstitial pneumonia (denoting IPF), fibrotic NSIP and a grouping of predominantly inflammatory disorders, seen in $45 \%, 35 \%$ and $20 \%$ of patients, were associated with 6-year survival rates of $10 \%, 50 \%$ and $100 \%$, respectively. It is difficult to envisage a more definitive prognostic separation. Thus, the application of the ATS/ERS system identifies separate patient subsets with survival rates broadly similar to those of patients with lung cancer, breast cancer and basal cell carcinoma of the skin. Thus far, cancer specialists have resisted the temptation to amalgamate these into "cryptogenic cancer". In diffuse lung disease, as in oncological practice, diagnostic distinctions can sometimes be difficult in individual patients, with interobserver and intraobserver diagnostic variation, ${ }^{9}{ }^{10}$ and diagnostic overlap between the classic entities, ${ }^{11}$ both recognised problems. However, even without good understanding of the entity with which one is dealing, diagnostic distinctions are worth pursuing because they allow informed discussion of treatment goals as well as accurate management. In idiopathic interstitial pneumonia, accurate diagnosis, which can often be based on appearances on HRCT rather than histopathological findings, distinguishes between inexorably progressive fibrosis (IPF), indolent fibrosis that sometimes has a good treated outcome (fibrotic NSIP) and the remaining inflammatory disorders that often do well with relatively restrained levels of treatment. To fail to make these distinctions is to play dice with patient management. "Presentation with CFA" is no longer an acceptable final diagnosis.
The counter-argument is that idiopathic interstitial pneumonias other than IPF are vanishingly rare in non-referral populations, and therefore have little or no relevance in routine practice. This contention, used to justify retention of the term "CFA" in its historical sense, is based on the more advanced age of nonreferral populations and the observation of a somewhat lower prevalence of NSIP in older patients in some series from specialist centres. However, the trend has not, in reality, been properly quantified and there is a pressing need for further studies of this question in unselected populations, perhaps based on appearances on HRCT rather than histopathological findings. The data of Rudd et $a l^{2}$ are instructive because they provide strong indirect evidence that a substantial minority of patients with "CFA" do, in fact, have disorders other than IPF. A response to treatment, as judged by marked increases in pulmonary function indices, was seen in $35 \%$ of patients, a figure that uncannily resembles the response rate reported in the histopathological study discussed earlier, ${ }^{8}$ in which $<50 \%$ of patients had IPF. By contrast, responsiveness seldom exceeds 10\% in studies of IPF in the post-ATS/ERS classification era The mortality data in the BTS study is also persuasive. Subsequent to the ATS ERS classification, outcomes of patients treated for IPF have been evaluated against age stratification in only one large cohort $^{12}$; in patients aged $\geqslant 70$, the 4-year survival of $15 \%$ is substantially lower than the 4-year survival of $40 \%$ in the BTS cohort. Taken together, the response rates and mortality data in the BTS study are highly suggestive of a large subgroup of patients with interstitial pneumonias other than IPF (including undiagnosed hypersensitivity pneumonia), who are not best served by an indiscriminate final diagnosis of "CFA".

It is unrealistic to hope to rehabilitate historical terms when a new classification is, in effect, written in marble-minor refinement aside, the ATS/ERS classification of the idiopathic interstitial pneumonias is here to stay. For clarity of thought, the term "CFA" should now be used as strictly synonymous with IPF, as currently defined using HRCT or biopsy criteria, in the correct clinical context. The creation of a new term such as "CFA clinical syndrome", for use by epidemiologists in studies of pulmonary fibrosis, is now absolutely necessary. It is time to move on.

Thorax 2007:62:3-4.

doi: $10.1136 /$ thx.2005.051011

\section{Authors' affiliations \\ A U Wells, D M Hansell, A G Nicholson, Royal Brompton Hospital, London, UK}

Correspondence to: $\operatorname{Dr} \mathrm{A} U$ Wells, Royal Brompton Hospital, Sydney Street, London SW3 6NP, UK; a.wells@rbh.nthames.nhs.uk

Competing interests: None declared.

\section{REFERENCES}

1 American Thoracic Society, European Respiratory Society. American Thoracic Society/European Respiratory Society international multidisciplinary consensus classification of the Idiopathic Interstitial Pneumonias. Am J Respir Crit Care Med 2002; 165:277-304

2 Rudd R, Prescott RJ, Chalmers JC, et al. British Thoracic Society Study of cryptogenic fibrosing alveolitis: response to treatment and survival. Thorax, this issue.

3 American Thoracic Society. Idiopathic pulmonary fibrosis: diagnosis and treatment. International consensus statement. Am J Respir Crit Care Med 2000;161:646-64.

4 Bjoraker JA, Ryu JH, Edwin MK, et al. Prognostic significance of histopathological subsets in idiopathic pulmonary fibrosis. Am J Respir Crit Care Med 1998; 157:199-203.

5 Johnston ID, Prescott RJ, Chalmers JC, et al. British Thoracic Society study of cryptogenic fibrosing alveolitis: current presentation and initial management. Fibrosing Alveolitis Subcommittee of the Research Committee of the British Thoracic Society. Thorax 1997;52:38-44.

6 Nagai S, Kitaichi $M$, Itoh $\mathrm{H}$, et al. Idiopathic nonspecific interstitial pneumonia/fibrosis: comparison with idiopathic pulmonary fibrosis and BOOP. Eur Respir J 1998;12:1010-19.

7 Selman M, Pardo A, Barrera L, et al. Gene expression profiles distinguish idiopathic pulmonary fibrosis from hypersensitivity pneumonitis. Am J Respir Crit Care Med 2006;173:188-98.

8 Nicholson AG, Colby TV, du Bois RM, et al. The prognostic significance of the histologic pattern of interstitial pneumonia in patients presenting with the clinical entity of cryptogenic fibrosing alveolitis. Am J Respir Crit Care Med 2000;162:2213-17.

9 Nicholson AG, Addis BJ, Bharucha $\mathrm{H}$, et al. Interobserver variation between pathologists in diffuse parenchymal lung disease. Thorax 2004:59:500-5.

10 Aziz ZA, Wells AU, Hansell DM, et al. HRCT diagnosis of diffuse parenchymal lung disease: inter-observer variation. Thorax 2004;59:506-11.

11 Flaherty KR, Thwaite EL, Kazerooni EA, et al. Radiological versus histological diagnosis in UIP and NSIP: survival implications. Thorax 2003;58:143-8.

12 King TE, Tooze JA, Schwarz MI, et al. Predicting survival in idiopathic pulmonary fibrosis: scoring system and survival model. Am J Respir Crit Care Med 2001; 164:1171-81. 


\section{Priorities for respiratory research in the UK}

\section{Stephen T Holgate, on behalf of the UK Respiratory Research Strategy Committee}

\section{Respiratory diseases are placing an increasing burden on the UK health system}

$\mathrm{n}$

ne past, respiratory research has proved itself effective in vanquishing major killers such as tuberculosis, transforming the lives of patients with asthma and developing life-saving noninvasive ventilation for those with chronic respiratory failure. Now, new problems affect our patients with respiratory diseases and present an enormous burden of ill health that we are currently ill equipped to deal with. The second edition of the Burden of lung disease was published by the British Thoracic Society in June 2006 ; it documents that respiratory diseases now kill one in five people in the UK, with the standardised mortality ratio for respiratory diseases showing a threefold difference across social classes. More people die from respiratory disease than from ischaemic heart disease. Respiratory diseases are the most common cause of long-term illness in children, result in the highest levels of consultations with general practitioners and are the second most common reason for emergency hospital admission. Examples of the severity of the situation from UK statistics are as follows:

- respiratory disease kills one in five people

- respiratory disease has the steepest socio-economic mortality gradient of any disease area

- respiratory disease is the most common reason for general practitioner consultation

- the only countries in Europe with a worse mortality rate from respiratory disease than the UK are Ireland, Malta, Kyrgyzstan, Tajikistan, Kazakhstan, Uzbekistan and the Republic of Moldova

- 5-year survival from lung cancer is $<8 \%$ (only pancreas is worse, 7000 cases/year)

- asthma is the most common chronic illness in children and pregnant women

- there are 10000 new cases of interstitial lung disease a year.

Although research has undoubtedly contributed to a greater understanding of disease mechanisms, epidemiology, diagnosis and treatment when compared with other specialities, progress has been slow. In the past 20 years, relative underfunding has meant that there has been less progress. One result of this is that the number of trainees pursuing a career in respiratory research has fallen to a dangerous level, and our ability to exploit the enormous diagnostic and treatment potential of recent advances in biology has been compromised.

This initiative is part of a process designed to change the situation. A broad coalition of groups with an interest in respiratory disease from childhood to old age has come together to develop a common list of priorities that identify those areas where more knowledge is urgently needed.

In October 2005, a large proportion of the respiratory researchers in the UK and those involved in supporting research (Medical Research Council, Wellcome Trust, Department of Health, British Lung Foundation and Asthma UK) and the professional societies (British Thoracic Society, British Association for Lung Research and the British Paediatric Respiratory Research Society) convened a workshop in which the current state of respiratory research was reviewed and possible mechanisms were discussed to consider how the capacity could be built. The first step was to identify the major priorities for research in the near future and then to bring together the various research funders to see how these could be used to help rebuild the capacity. A further highly interactive workshop involving both researchers and funders led to a consensus over those areas where initial effort should be focused. The seven themes that emerged are presented, not in any order of priority.

\section{EARLY LIFE EVENTS: DEVELOPMENT AND LUNG AGEING}

Interactions between maturation of immune function and lung growth contribute to the development of airway disease. Both are subject to genetic control, and recent interest has focused on genes that regulate lung and airway development. The premise that host response to microbial stimuli has altered as a result of changes in the nature, timing or dose of the signals received has gained much attention, but there remains the possibility that changes in the host response are secondary to programming of the immune system by early environmental influences. Two specific areas of interest are the role of pattern recognition (eg, Toll-like receptors and regulatory $\mathrm{T}$ cells) in generating antiviral responses in the respiratory tract, and the occurrence and relevance of airway injury and remodelling to disease. One of the greatest challenges will be to identify, with confidence, those infants who have or will go on to develop airway disease in later childhood or adulthood. Several obstacles are yet to be overcome. We know relatively little about the natural history of the intrauterine and immediate postnatal development of the respiratory tract and immune system. Access to peripheral blood or biopsy specimens is severely limited. Measurement of lung function in the very young is also difficult. The ideal technique should have easily transferable technology that is cost effective so that large numbers of children can be studied. Liaison with engineers and nano technologists will be helpful to develop novel approaches. Lung diseases may develop, persist, remit or relapse during adolescence and adulthood, and decline in lung function in adults may be mediated by early life events, but the determination of these outcomes is poorly understood. We need to study the interplay of genetic and early environmental factors that influence the severity of asthma and cystic fibrosis and increased susceptibility to chronic obstructive pulmonary disease (COPD). Changes in the immune system and host response with increasing age may predispose to malignancy and pulmonary fibrosis. Declining lung physiology may precipitate disease, exacerbate existing disease or contribute to disease in other systems (eg, sleep disordered breathing, cardiovascular disease and neurological decline). An improved understanding of development and remission should lead to opportunities for prevention. A better understanding of age-related responses to pharmacotherapy may improve treatment. Key research areas include the natural history of early development of the respiratory tract and immune system and the techniques needed to understand normal airway growth, development and decline in health and disease. It is important to define the normal ontogeny of Toll-like receptors and immunoregulatory $\mathrm{T}$ cell function and how this changes 
with disease. A prospective birth cohort using an appropriate intervention strategy and including a placebo group could provide an opportunity to do this. For lung disease in adolescence and adulthood, there is a need to identify the factors influencing development, remission, relapse, progression and severity of airway disease, and with this understanding whether the natural history of lung disease can be modified beneficially by early life interventions.

\section{AIRWAY DISEASE AND SLEEP APNOEA SYNDROMES}

Asthma, COPD and sleep apnoea syndromes have major health consequences and socioeconomic effects. Each of these diseases is common and constitutes a high proportion of the total respiratory disease burden in the UK. On the basis of recent national consultations, there are major areas where further research could enhance our understanding of the cause, prevention and treatment of disease. Advances in technologies ranging from molecular to whole-body approaches create unique opportunities to make substantial advances in knowledge of these disorders. Key research questions include identifying opportunities for the primary and secondary prevention of acute exacerbations and chronicity of lower airway diseases, the identification of genetic and epigenetic-environmental interactions in susceptibility to all three disorders, the application of pharmacogenetic approaches to improve clinical care and the application of non-invasive imaging and physiological techniques to ascertain normal ontogeny, perturbations in disease and improvement with treatment. These could include the development of technologies for assessing intrauterine development of the lung and immune system, measurement of lung functions from birth to old age, assessment of different lung compartments including airways, parenchymal and vascular structures, and involvement of the central and peripheral nervous system. In relation to the application of new technologies and impact evaluation, there is a requirement to identify unmet clinical needs and for targeted preventive and therapeutic strategies, including palliative care for end-stage disease, accompanied by appropriate outcome measures to evaluate interventions.

\section{RESPIRATORY INFECTIONS}

Respiratory infections (excluding tuberculosis) account for $>6 \%$ of the global burden of human disease, causing more morbidity and mortality than all cancers. Poverty is associated with a $>20$-fold increase in the relative burden of lung infections, which disproportionately affect the very young and very old. In the USA, more is spent for research on small pox (US\$324 million in 2004) than on lung infections (US\$287 million); lung infections receive only one tenth of the funding provided for HIV/AIDS, which causes a similar disease burden. ${ }^{2}$

More infections are spread via the respiratory tract than by any other route. Respiratory pathogens are responsible for a huge worldwide disease burden; they mutate fast, cross species barriers, threaten patients with pre-existing lung diseases, evade antimicrobial treatments and respond rapidly to demographic and economic change. Some (eg, tuberculosis) are on the rise, whereas others (eg, severe acute respiratory syndrome and "avian" influenza) are emerging threats to health and prosperity. There is an urgent need for new vaccines and antimicrobial drugs, and for agents that beneficially modify host immune responses. We need evidence-based approaches to antimicrobial prescribing and to identify situations where combinations of drugs are required. Unnecessary antimicrobial use has led to major problems of antimicrobial resistance, side effects, patient dependence and costs, especially in the community. Appropriate, rational antimicrobial treatments are needed for patients with mild infections. Key research areas include the early "innate" response to microbial invasion, the influence of these responses on $\mathrm{T}$ cell and $\mathrm{B}$ cell immunity, the role of overexuberant immunity in causing disease and studies of immune evasion, reinfection and persistence. Other important topics include the effects of genetic variation, infection history, coinfections, immune immaturity and senescence. We need a better understanding of the role of infections in chronic inflammatory diseases.

\section{LUNG CANCER}

Lung cancer is the most common cause of death from cancer for both men and women in the UK. Its incidence is still rising among women in Britain and will reach epidemic proportions in the Far East, as well as continue to rise in many European countries for years to come. The major cause, tobacco smoking, is well established, and efforts continue to educate populations on the dangers of smoking. This has had success, and, in the USA, there are more lung cancers arising in exsmokers than in current smokers. Exposure to environmental tobacco smoke is also linked to increased cancer, and the success of campaigns to limit smoking in public places is a real achievement.

An urgent need prevails to understand the genetic and environmental factors for susceptibility to lung cancer, as only a small percentage of heavy smokers contract the disease; the genetic predisposition to lung cancer has only just begun to be studied. The late presentation of most patients has made curative treatment difficult, and studies looking at prognostic symptoms and signs at presentation have been unhelpful. Treatment of advanced disease presentation (the great majority of cases) is unlikely to have much of an impact in the near future. Key research areas include genetic and epigenetic studies of susceptibility and progression; an understanding of how to modify or halt tumour growth, and to identify potential inhibitors of growth; the application of improved technology to identify cancers earlier and at a better stage for potential cure; improve staging of known disease to optimise current available treatment; and evaluation of the potential of screening high-risk populations. It is starkly obvious that current treatments are totally inadequate.

\section{LUNG INJURY, REPAIR AND REGENERATION}

The lung is uniquely vulnerable to injurious agents both from the environment, because it exposes a large surface area to the air, and from within the body, because the pulmonary vascular bed receives the entire cardiac output. Tissue injury and the resulting inflammation and repair are processes that underlie most lung diseases. Although the past two decades have witnessed considerable advances in understanding these processes in disease pathogenesis, the mechanisms involved in disease chronicity that are amenable to intervention are largely unknown. Many lung diseases involve complex combinations of epithelial injury, vascular responses and aberrant repair, often in combination with different types of inflammation. For most chronic lung diseases, some understanding has been gained of the cellular and molecular mechanisms underlying disease pathogenesis, but often this knowledge is incomplete and "downstream" of the initiation by noxious agents. The recent rapid development of new in vitro technologies and integrative biological approaches will greatly facilitate future mechanistic studies. Key research areas include the application of cell and molecular approaches as well as genomics, proteomics and metabolomics to identify underlying mechanisms of lung injury, inflammation and repair responses, with a focus on understanding disease chronicity and tissue remodelling. The role of embryonic and adult stem cells in lung repair and regeneration is an important area for study. There is a need to apply 
newer technologies to identify biomarkers of chronic disease and acute exacerbations, and to apply these to epidemiological studies to gain a greater understanding of how environmental and other factors can influence the natural history and disease prognosis. There is an important requirement for improved animal models that better reflect chronic lung disease in humans and that can be applied to drug discovery.

\section{PHENOTYPING RESPIRATORY DISEASES}

The heterogeneity in clinical features, pathogenic processes and responses to treatment between individuals with respiratory disease is likely to be explained largely by distinct phenotypes that develop because of complex interactions between genetic and environmental factors. In the past, the classification of respiratory disease was largely descriptive, based on symptoms, signs and general functional abnormalities that grouped together individuals with diverse phenotypes. In the future, the accurate phenotyping of disease will have a major effect on the management of respiratory conditions and will lead to the ultimate cure of specific diseases.

The emergence of new investigative techniques, including genomics, proteomics and non-invasive imaging, offers exciting opportunities to classify respiratory diseases more accurately into distinct phenotypes. This approach will help in understanding the pathogenic processes that lead to the initiation and progression of disease, as well as to the development of new targeted treatments. Accurate phenotyping is also important for epidemiological studies of causation, which could lead to prevention. Advances in the identification of specific phenotypes are already being made in several respiratory diseases. Key research areas include determining which respiratory disease phenotypes are important, how they are identified in clinical practice, and their implications for susceptibility, prevention, diagnosis, treatment and prognosis of disease.

\section{DELIVERY OF CARE FOR LUNG DISEASE}

Eight million people in the UK have respiratory disease. The medical management of these patients is delivered in primary, secondary and tertiary care by consultants (who may be respiratory physicians or specialists in other fields), by hospital and community-based respiratory nurse specialists or practitioners, by general practitioners (a minority of whom may have a special interest in respiratory medicine), by practice-based nurses and healthcare assistants, and in future by medical care practitioners. There are also valuable contributions from clinical scientists, pharmacists, physiological measurement technicians, respiratory physiotherapists and others. How this care is delivered and in which sector of the health service is subject to only sporadic evaluation, with evaluation often being undertaken by enthusiasts and with results that cannot always be extrapolated elsewhere. Unlike other specialities, respiratory medicine encompasses a diverse range of disorders. Although some are infectious, short term and self-limiting, most patients with respiratory disorders have long-term non-communicable conditions. Management of those with longterm and disabling conditions should recognise the dominant role of self-care but all too often our patients are not given the skills, training and education they need to effectively self-manage their conditions. Rigorous methods to determine patient preferences, such as discrete choice experiments, are rarely used and benchmarking has not been optimally used, especially in conjunction with standards defined in guidelines.

Key research areas include the rigorous study of outcomes for care delivered in different arenas by different healthcare professionals and by lay people, with such evaluations including full health economic evaluation and benchmarking and educational requirements for healthcare providers (eg, outreach specialist clinics, use of lay educators, nurse prescribing, handover clinics for adolescents with lung disease, nurse practitioner clinics, the role of medical care practitioners). This area is particularly important in long-term respiratory disorders, especially those affecting elderly people, who often also have comorbidities. A further important focus for future research is the rigorous study of patient preferences utilising, for example, discrete choice experiments, especially for long-term disorders where self-care is vital. This should include factors that influence adherence to treatment, such as social needs. There is need for more research to enhance the accurate diagnosis of diverse respiratory diseases, many of which share symptoms with disorders of other systems. Evaluations of one-stop diagnostic centres and population studies are needed to define accurately the true burden and trends of respiratory illness in the UK. This could be achieved by the Health Survey of England maintaining a focus on respiratory disease, and by monitoring quality and outcomes framework data. For rarer respiratory disorders, continuation and extension of the British Thoracic Society Orphan (Rare) Lung Disease project would be desirable.

Strong social class and ethnic inequalities are associated with some lung diseases (eg, lung cancer, asthma, tuberculosis and COPD). Research is needed to determine how best to reduce these differentials and to reduce inequalities in smoking cessation. Some respiratory conditions are rare, entail high costs and need specialist management. Many others are managed in the community, and there should be a focus on respiratory networks to ensure an optimal research environment to answer questions on the prevention of respiratory disease and optimal management. Rigorous evaluation of the use of new technologies for diagnostic and monitoring purposes is needed-for example, exhaled nitric oxide, telephone and email consultations, and e-learning. Excellent guidelines exist for most respiratory conditions, but implementation is patchy. Evaluations of optimal methods of teaching the correct management of respiratory illness are needed.

By focusing a multidisciplinary effort involving researchers across the full range of respiratory medicine and healthcare, and combining resources from different funders, hopefully, the poor state of research in this major field of unmet clinical need can be addressed, starting with the rebuilding of capacity. A cadre of new young researchers to drive forward understanding and healthcare in lung disease will provide a platform for the subsequent development of this neglected area of human illness. Although this was a UK initiative, we believe that the conclusions drawn are applicable to lung disease in other countries.

Thorax 2007;62:5-7.

doi: 10.1136/thx.2006.073882

Correspondence to: Professor Stephen T Holgate, Southampton General Hospital, Southampton, Hants SO16 6YD, UK; sth@soton.ac.uk

Received 23 October 2006

Accepted 31 October 2006

Competing interests: None declared.

\section{REFERENCES}

1 British Thoracic Society. Burden of lung disease, 2nd edn. London: British Thoracic Society 2006. 2 Mizgerd JP. Lung infection-a public health priority. PloS Med 2006;3:e76. 